\title{
Pseudoaneurysm of the right ventricular outflow tract: diagnosis by colour flow mapping
}

\author{
N Sreeram, G R Sutherland, J McGhie
}

\begin{abstract}
Eleven years after surgical repair of pulmonary atresia with a ventricular septal defect with a valved conduit, a 20 year old patient was diagnosed on follow up echocardiography to have a right ventricular pseudoaneurysm related to the conduit. The echocardiographic findings were confirmed at reoperation and the pseudoaneurysm was excised.
\end{abstract}

A pseudoaneurysm of the right ventricle or right ventricular outflow tract is rare. Most reported cases presented either immediately after or within a few months of a right ventriculotomy. ${ }^{12}$ The diagnosis in every reported case was confirmed by angiography or at necropsy. Pseudoaneurysms of the left ventricle are best defined by colour flow mapping. ${ }^{3}$ We describe what we believe to be the first case of a right ventricular pseudoaneurysm in which the initial diagnosis was made by colour flow mapping.

\section{Case report}

At the age of nine this 20 year old woman had had complete repair of pulmonary atresia with ventricular septal defect. At operation the ventricular septal defect was closed and a valved conduit containing a $20 \mathrm{~mm}$ Hancock valve was inserted between the right ventricle and the main pulmonary artery. Routine cardiac catheterisation four years after operation showed a severely stenotic Hancock valve within a calcified conduit (right ventricle to pulmonary artery peak systolic gradient $100 \mathrm{~mm} \mathrm{Hg}$ ). Because the patient was symptom free at that time no further operation was contemplated. She remained symptom free over the next seven years until mild tiredness and intermittent sharp retrosternal pain unrelated to exercise developed.

Clinical examination showed no new signs. A prominent right ventricular impulse was palpable. An ejection systolic and early diastolic murmur (present seven years before) was heard in the pulmonary area. The chest $x$ ray showed mild cardiomegaly. However, a striking new radiological abnormality was widening of the cardiac silhouette by a globular shadow in the region of the main pulmonary artery (fig 1). The electrocardiogram showed first degree atrioventricular block, a right bundle branch block pattern, and occasional atrial and ventricular extrasystoles. Cross sectional echocardiography showed severe right ventricular hypertrophy with abnormal motion of the interventricular septum. A large echo-free space was noted behind the thoracic wall; this was in front of and to the left of the right ventricular outflow tract. Imaging alone did not initially show a communication between this space and the right ventricle. Continuous wave Doppler examination confirmed a severely stenotic Hancock valve with peak systolic velocity in the conduit of $5 \mathrm{~m} / \mathrm{s}$. No flow was recorded within the echo-free space by pulsed Doppler. Colour flow mapping confirmed low velocity flow within this space. Detailed examination showed that this space communicated with the right ventricle through a narrow ostium sited beneath the Hancock valve (as shown by the colour flow map), confirming the diagnosis of pseudoaneurysm. Colour flow mapping also showed multiphasic turbulent flow across the ostium throughout the cardiac cycle (fig 2). Continuous and pulsed wave Doppler did not provide additional information: despite visualisation of the ostium, the peak velocities of flow could not be recorded because of the large angle between the ultrasound beam and the direction of flow as seen on the colour flow map. Bacterial endocarditis was excluded as a cause of the development of the pseudoaneurysm by repeated blood cultures, which were sterile.

Despite the unequivocal diagnosis by colour flow mapping, cardiac catheterisation was performed because the lesion is so rare. The right ventricular angiogram showed faint opacification of the pseudoaneurysm with contrast, presumably because it was large and the volume flowing to and fro during each cardiac cycle was low; no entry or exit point could be defined. The echocardiographic findings were confirmed at operation; the pseudoaneurysm was excised and the Hancock conduit replaced.

\section{Discussion}

A pseudoaneurysm results from a rupture of the ventricular wall with containment of the resultant haematoma by pericardium or extracardiac structures. Left ventricular pseudoaneurysms are known to occur after events such as myocardial infarction, ven- 

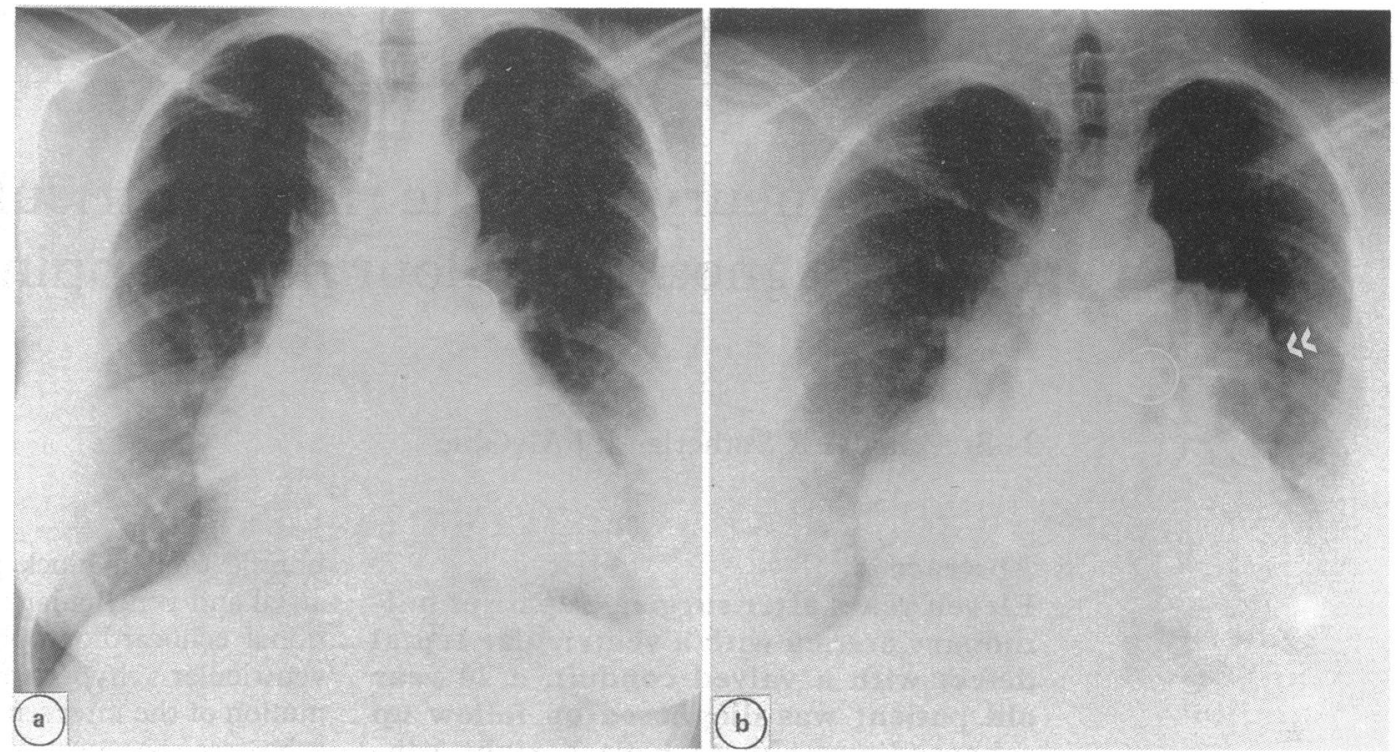

Figure 1 Chest $x$ ray (a) showing the appearance four years before. The prosthetic valve ring was in the pulmonary position and there was mild cardiomegaly. (b) A new globular shadow along the left border of the cardiac silhouette (arrows) in the region of the main pulmonary artery.

triculotomy, chest trauma, infective endocarditis, or inflammatory processes affecting the heart. ${ }^{4}$ Right ventricular pseudoaneurysms are less well described, and most of the few reported cases occurred within a few days or months of cardiac surgery, and were probably caused by surgical sequelae or endocarditis. Our patient had none of the above risk factors. In particular there was no evidence from the history or from subsequent investigations for bacterial endocarditis.

Left ventricular pseudoaneurysms are known to rupture even in the chronic stage. When right ventricular pressures are high, the risk of a rupture of right ventricular pseudoaneurysm may be similar. This, and the possibility of a successful outcome after surgical resection, means that prompt recognition of this lesion is important. ${ }^{5}$ Angiocardiography is not without risk and is sometimes less than optimal. ${ }^{6}$ In our patient the right ventricular angiogram failed adequately to opacify the pseudoaneurysm or show its ostium. The cross sectional features of pseudoaneurysm at echocardiography are well described; they include the demonstration of a saccular or globular aneurysmal chamber, a discontinuity of the endocardial image at the site of communication with the pseudoaneurysm, and a narrow orifice

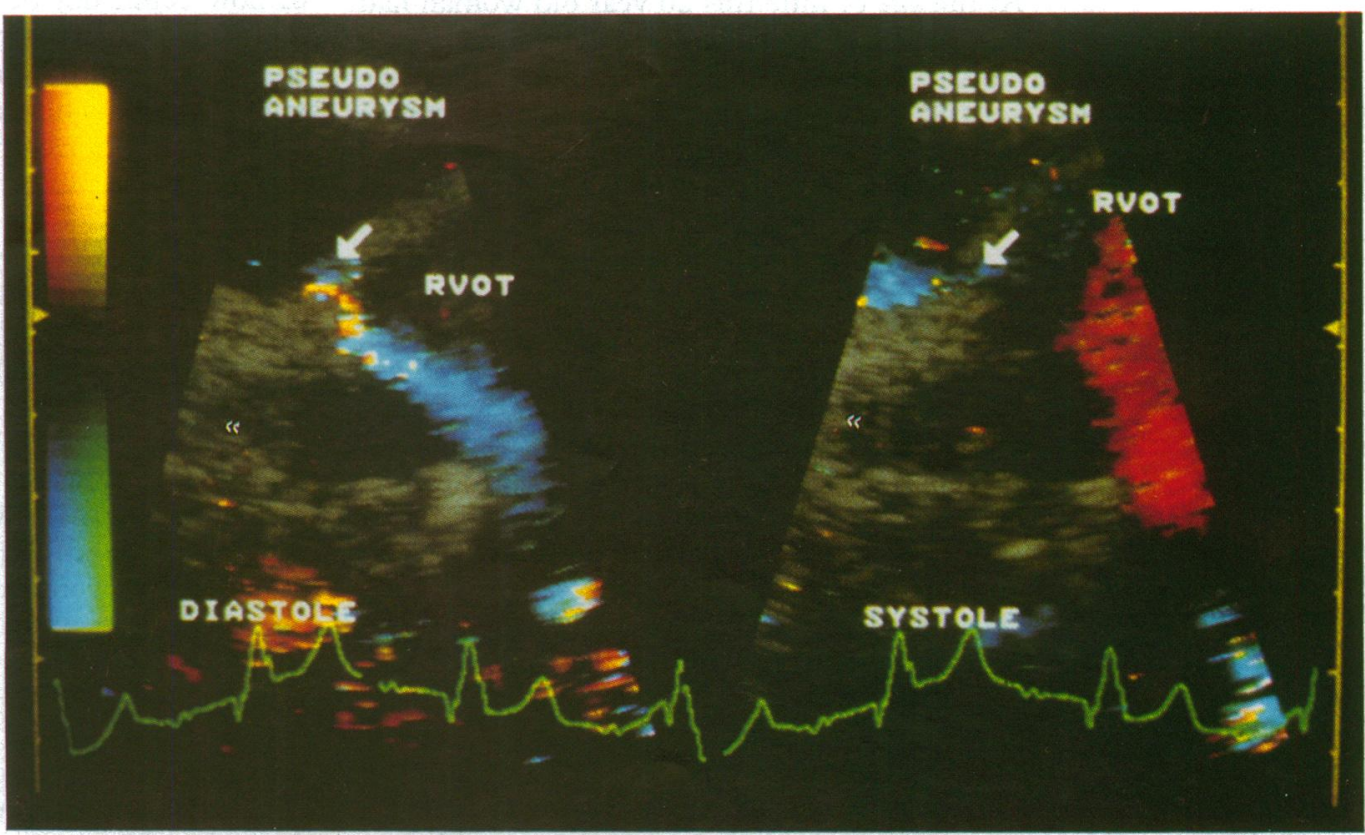

Figure 2 Cross sectional echocardiogram in the high parasternal view. Doppler colour flow map showing multiphasic flow into and out of the right ventricular pseudoaneurysm in different phases of the cardiac cycle (arrow). The pseudoaneurysm is represented by the large echo-free space at the top of the sector. The left hand panel shows diastolic flow from the pseudoaneurysm (arrow) in systole. The position of the prosthetic valve is indicated by the smaller double arrows. RVOT, right ventricular outflow tract. 
diameter compared with the diameter of the aneurysm. ${ }^{7}$ The diagnosis, however, hinges on the ability to show the ostium of the pseudoaneurysm. Pulsed and continuous wave Doppler may then be used to show turbulent high velocity flow through the restrictive ostium. Neither of these techniques proved helpful in our patient. A definite diagnosis was made only after colour flow mapping showed flow within the anterior echo-free space and identified the narrow communication between the right ventricular outflow tract and this space. The characteristic multiphasic pattern of turbulent flow described for left ventricular pseudoaneurysm was also noted in this case. ${ }^{3}$ We believe that colour flow mapping may be the safest and most accurate investigative technique for diagnosing this rare lesion.
N S is supported by a British-Dutch fellowship of the British Heart Foundation.

1 Campbell M, Deuchar DC, Brock R. Results of pulmonary valvotomy and infundibular resection in 100 cases of Fallot's tetralogy. $\mathrm{Br}$ Med J 1954;ii:111-22.

2 McCord MC, Blount SG. Complications following infundibular resection in Fallot's tetralogy. Circulation 1955;11:754-60.

3 Sutherland GR, Smyllie JS, Roelandt JRTC. Advantages of colour flow imaging in the diagnosis of left ventricular pseudoaneurysm. Br Heart J 1989;61:59-64.

4 Davidson KH, Parisi AF, Harrington JJ, Barsamian EM, Fishbein MC. Pseudoaneurysm of the left ventricle: an unusual echocardiographic presentation. Ann Intern Med 1977;86:430-3.

5 Vlodaver Z, Coe JI, Edwards JE. True and false left ventricular aneurysms. Propensity for the latter to rupture. Circulation 1975;51:567-72.

6 Gatewood RP, Nanda NC. Differentiation of left ventricular pseudoaneurysm from true aneurysm with two dimensional echocardiography. Am J Cardiol 1980;46:869-78

7 Catherwood E, Mintz GS, Kotler MN, Parry WR, Segal BL. Two-dimensional echocardiographic recognition of left ventricular pseudoaneurysm. Circulation 1980;62: 294-303. 